\title{
Fast Computation of Electromagnetic Wave Propagation and Scattering for Quasi-cylindrical Geometry
}

\author{
Shaolin Liao \\ Electrical and Computer Engineering, 1415 Engineering Drive, Univ. of Wisconsin, Madison, U.S.A., 53706
}

\begin{abstract}
The cylindrical Taylor Interpolation through FFT (TI-FFT) algorithm for computation of the near-field and far-field in the quasi-cylindrical geometry has been introduced. The modal expansion coefficient of the vector potentials $\mathbf{F}$ and $\mathbf{A}$ within the context of the cylindrical harmonics (TE and TM modes) can be expressed in the closed-form expression through the cylindrical addition theorem. For the quasi-cylindrical geometry, the modal expansion coefficient can be evaluated through FFT with the help of the Taylor Interpolation (TI) technique. The nearfield on any arbitrary cylindrical surface can be obtained through the Inverse Fourier Transform (IFT). The far-field can be obtained through the Near-Field Far-Field (NF-FF) transform. The

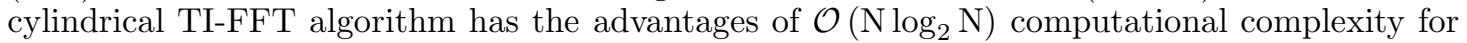
$\mathrm{N}=\mathrm{N}_{\phi} \times \mathrm{N}_{z}$ computational grid, small sampling rate (large sampling spacing) and no singularity problem.
\end{abstract}

\section{INTRODUCTION}

The planar Taylor Interpolation through FFT (TI-FFT) algorithm introduced before [1] has been shown to be efficient in the computation of narrow-band beam propagation and scattering for the quasi-planar geometry [2, 3, 4, 5, 6, 7, 8, 9, 10, 11, 12, 13, 14, 15, 16, 17, 18. However, cylinder-like geometry is not uncommon in the electromagnetic engineering, e.g., the input mirror system design 14 for the high-power gyrotron application. In such case, the planar TI-FFT algorithm is not efficient and we have developed the cylindrical TI-FFT to solve the problem.

For the cylindrical geometry, the computation is efficient because the electromagnetic field that is expressed in the cylindrical harmonics can be numerically implemented through the FFT. For the quasi-cylindrical geometry, the FFT can still be used, with the help of the Taylor Interpolation (TI) technique. Fig. 1 shows the scheme used to illustrate the cylindrical TI-FFT algorithm and the time dependence $e^{i \omega t}(i \equiv \sqrt{-1})$ is used in this article.

\section{THE NEAR-FIELD AND THE FAR-FIELD}

In this section, the near-field and the far-field for surface currents $\left(\mathbf{M}_{s}, \mathbf{J}_{s}\right)$ are presented within the context of the cylindrical harmonics.

\section{The Near-field}

It can be shown [8] that the vector potential $(\mathbf{F}, \mathbf{A})$ due to surface currents $\left(\mathbf{M}_{s}, \mathbf{J}_{s}\right)$ for the scattering phenomenon in the region $\rho>\rho^{\prime}$ can be expressed as

$$
\begin{gathered}
\begin{array}{c}
\mathbf{F}(\mathbf{r}) \\
\mathbf{A}(\mathbf{r})
\end{array}=\operatorname{IFT}\left\{\begin{array}{cc}
\mathbf{f}_{m}^{h} & \left.H_{m}^{(2)}(\Lambda \rho)\right\}, \\
\mathbf{g}_{m}^{h} &
\end{array}\right. \\
\mathbf{f}_{m}^{h}=\frac{1}{i 4} \iint_{S} d S^{\prime} \quad \begin{array}{l}
\epsilon \mathbf{M}_{s}\left(\mathbf{r}^{\prime}\right) \\
\mathbf{g}_{m}\left(\mathbf{r}^{\prime}\right)
\end{array} H_{m}^{(1)}(\Lambda \rho) e^{i m \phi^{\prime}} e^{i h z^{\prime}},
\end{gathered}
$$

where $H_{m}^{(1)}(\cdot)$ and $H_{m}^{(2)}(\cdot)$ are Hankel functions of the first kind and the second kind of integer order $m$ respectively. The Inverse Fourier Transform (IFT) has been defined as,

$$
\operatorname{IFT}\{\cdot\}=\frac{1}{2 \pi} \sum_{m=-\infty}^{\infty} \int_{-\infty}^{\infty} d h\{\cdot\} e^{-i m \phi} e^{-i h z} .
$$




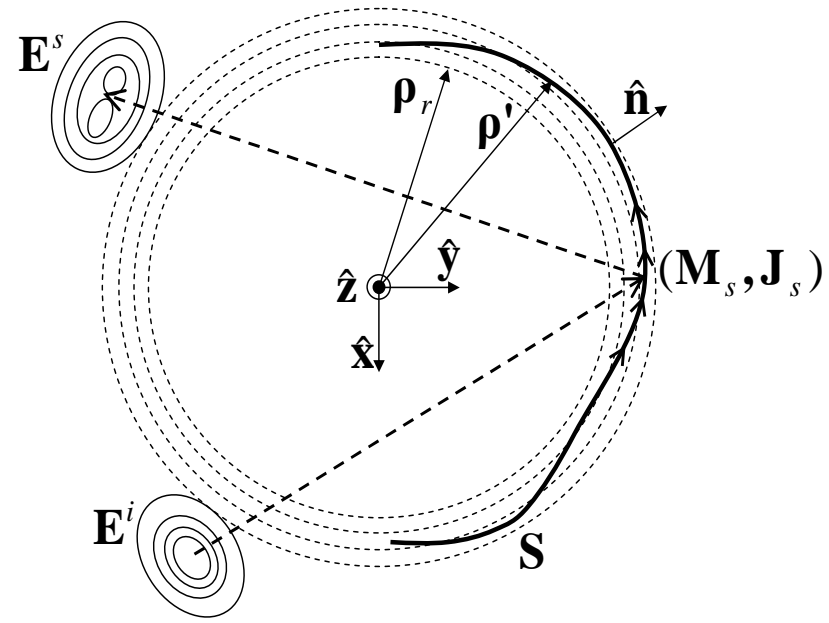

Figure 1: The scattering of the narrow-band beam: the incident field $\mathbf{E}^{i}$ propagates onto PEC surface $S$ and is back-scattered to $\mathbf{E}^{s}$. The induced surface currents $\left(\mathbf{M}_{s}, \mathbf{J}_{s}\right)$ can be obtained through the Method of Moment (MoM) or Physical Optics (PO) approximation if PEC surface $S$ is smooth enough. $\rho^{\prime}$ is the source coordinate and $\rho_{r}$ is the radius of the reference cylindrical surface. $\hat{\mathbf{n}}$ is the surface normal to $S$.

The electromagnetic field $(\mathbf{E}, \mathbf{H})$ is given as

$$
\begin{aligned}
& \mathbf{E}(\mathbf{r})=-\frac{1}{\epsilon} \nabla \times \mathbf{F}(\mathbf{r})-i \omega \mathbf{A}(\mathbf{r})+\frac{1}{i \omega \epsilon \mu} \nabla^{\prime}\left[\nabla^{\prime} \cdot \mathbf{A}(\mathbf{r})\right] . \\
& \mathbf{H}(\mathbf{r})=\frac{1}{\mu} \nabla \times \mathbf{A}(\mathbf{r})-i \omega \mathbf{F}(\mathbf{r})+\frac{1}{i \omega \epsilon \mu} \nabla^{\prime}\left[\nabla^{\prime} \cdot \mathbf{F}(\mathbf{r})\right] .
\end{aligned}
$$

\section{The Cylindrical Harmonics}

The cylindrical TE and TM modes are obtained when the magnetic (electric) surface current has only $\hat{\mathbf{z}}$-component, i.e., $\mathbf{M}_{s}=\hat{\mathbf{z}} \mathrm{M}_{s, z}\left(\mathbf{J}_{s}=\hat{\mathbf{z}} \mathrm{J}_{s, z}\right)$. From (11)-(15),

$$
\begin{gathered}
\mathbf{M}_{m}^{h}(\mathbf{r})=\left[\hat{\boldsymbol{\rho}} \frac{m}{i \rho} H_{m}^{(2)}(\Lambda \rho)-\hat{\boldsymbol{\phi}} \Lambda \frac{\partial H_{m}^{(2)}(\Lambda \rho)}{\partial(\Lambda \rho)}\right] e^{-i m \phi} e^{-i h z} . \\
\mathbf{N}_{m}^{h}(\mathbf{r})=\left[\hat{\boldsymbol{\rho}} \frac{h \Lambda}{i k} \frac{\partial H_{m}^{(2)}(\Lambda \rho)}{\partial(\Lambda \rho)}-\hat{\boldsymbol{\phi}} \frac{m h}{k \rho} H_{m}^{(2)}(\Lambda \rho)+\hat{\mathbf{z}} \frac{\Lambda^{2}}{k} H_{m}^{(2)}(\Lambda \rho)\right] e^{-i m \phi} e^{-i h z} .
\end{gathered}
$$

The electromagnetic field $(\mathbf{E}, \mathbf{H})$ can be expressed as the combination of the TE and TM modes,

$$
\begin{gathered}
\mathbf{E}(\rho)=\sum_{m}\left\{\int_{-\infty}^{\infty}\left[a_{m}^{h} \mathbf{M}_{m}^{h}(\rho)+b_{m}^{h} \mathbf{N}_{m}^{h}(\rho)\right] d h\right\}, \\
\mathbf{H}(\rho)=\frac{i}{\eta} \sum_{m}\left\{\int_{-\infty}^{\infty}\left[a_{m}^{h} \mathbf{N}_{m}^{h}(\rho)+b_{m}^{h} \mathbf{M}_{m}^{h}(\rho)\right] d h\right\}, \\
a_{m}^{h}=-\frac{1}{2 \pi \epsilon} \mathrm{f}_{m, z}^{h}, \quad b_{m}^{h}=-\frac{i v}{2 \pi} \mathrm{g}_{m, z}^{h},
\end{gathered}
$$

where $\eta=\sqrt{\frac{\mu}{\epsilon}}$ and $v=\frac{1}{\sqrt{\mu \epsilon}}$ is the electromagnetic wave velocity in the homogeneous medium. 


\section{The Far-field}

The far-field can be obtained through the Near-Field Far-Field (NF-FF) transform [19],

$$
\begin{aligned}
& \mathbf{E}(\mathbf{R})=-\frac{2 k \sin \theta e^{-i k R}}{R} \sum_{m} i^{m} e^{-i m \phi}\left[\hat{\boldsymbol{\phi}} a_{m}^{h}+\hat{\boldsymbol{\theta}} i b_{m}^{h}\right], \\
& \mathbf{H}(\mathbf{R})=-\frac{2 k \sin \theta e^{-i k R}}{\eta R} \sum_{m} i^{m} e^{-i m \phi}\left[\hat{\boldsymbol{\phi}} i b_{m}^{h}-\hat{\boldsymbol{\theta}} a_{m}^{h}\right],
\end{aligned}
$$

where $\mathbf{R}$ is the coordinate in the far-field and $R=|\mathbf{R}|$.

\section{THE CYLINDRICAL TI-FFT ALGORITHM}

For the narrow-band beam and the quasi-cylindrical surface, both the electromagnetic field in (81)-(9) and the modal expansion coefficient in (2) can be expressed in the Taylor series, which facilitates the use of FFT. Due to the similarity, only TE mode will be considered in this article.

\section{The Electromagnetic Field}

Generally, the near-field $\mathbf{E}$ can be expressed in the Taylor series,

$$
\mathbf{E}\left(\rho_{r}+\delta \rho\right)=\mathbf{E}\left(\rho_{r}\right)+\left.\sum_{n=1}^{\infty} \frac{1}{n !} \frac{\partial^{(n)} \mathbf{E}}{\partial \rho^{(n)}}\right|_{\rho_{r}}(\delta \rho)^{n}
$$

where, $\rho_{r}$ is the reference cylindrical surface and the Taylor coefficient $\left.\frac{\partial^{(n)} \mathbf{E}}{\partial \rho^{(n)}}\right|_{\rho_{r}}$ can be expressed in the form of IFT. Take TE mode $\left(\mathbf{M}_{m}^{h}\right)$ as an example, for $\hat{\boldsymbol{\phi}}$-component $\mathrm{E}_{\phi}$, from ([6) and (8),

$$
\mathrm{E}_{\phi}(\rho)=\operatorname{IFT}\left\{\frac{\Lambda}{\epsilon} \frac{\partial H_{m}^{(2)}(\Lambda \rho)}{\partial(\Lambda \rho)} \mathrm{f}_{m, z}^{h}\right\}
$$

Now, the Taylor coefficient for $\mathrm{E}_{\phi}(\rho)$ are given as

$$
\left.\frac{\partial^{(n)} \mathbf{E}}{\partial \rho^{(n)}}\right|_{\rho_{r}}=\operatorname{IFT}\left\{\left.\frac{\Lambda^{n+1}}{\epsilon} \frac{\partial^{(n+1)} H_{m}^{(2)}(\Lambda \rho)}{\partial(\Lambda \rho)^{(n+1)}}\right|_{\rho_{r}} \mathrm{f}_{m, z}^{h}\right\} .
$$

Similar argument holds for other electromagnetic field components and TM mode.

\section{The Modal Expansion Coefficient}

Similarly, $H_{m}^{(1)}\left(\Lambda \rho^{\prime}\right)$ in the the modal expansion coefficients $\left(\mathbf{f}_{m}^{h}, \mathbf{g}_{m}^{h}\right)$ in (2) can be expanded into the Taylor series,

$$
H_{m}^{(1)}\left(\Lambda\left[\rho_{r}+\delta \rho^{\prime}\right]\right)=H_{m}^{(1)}\left(\Lambda \rho_{r}\right)+\left.\sum_{n=1}^{\infty} \frac{\Lambda^{n}}{n !} \frac{\partial^{(n)} H_{m}^{(1)}(\Lambda \rho)}{\partial(\Lambda \rho)^{(n)}}\right|_{\rho_{r}}\left(\delta \rho^{\prime}\right)^{n}
$$

where $\delta \rho^{\prime}=\rho^{\prime}-\rho_{r}$. Now, the modal expansion coefficient in (2) is given as,

$$
\mathbf{f}_{m}^{h}=\left.\sum_{\triangle S} \sum_{n=0}^{\infty} \operatorname{FT}\left\{\gamma_{m}\left(\Lambda \rho_{r}\right) \underset{\mu \tilde{\mathbf{J}}_{s}\left(\mathbf{r}^{\prime}\right)}{\epsilon \tilde{\mathbf{M}}_{s}\left(\mathbf{r}^{\prime}\right)}\left(\delta \rho^{\prime}\right)^{n}\right\}\right|_{\triangle S}
$$




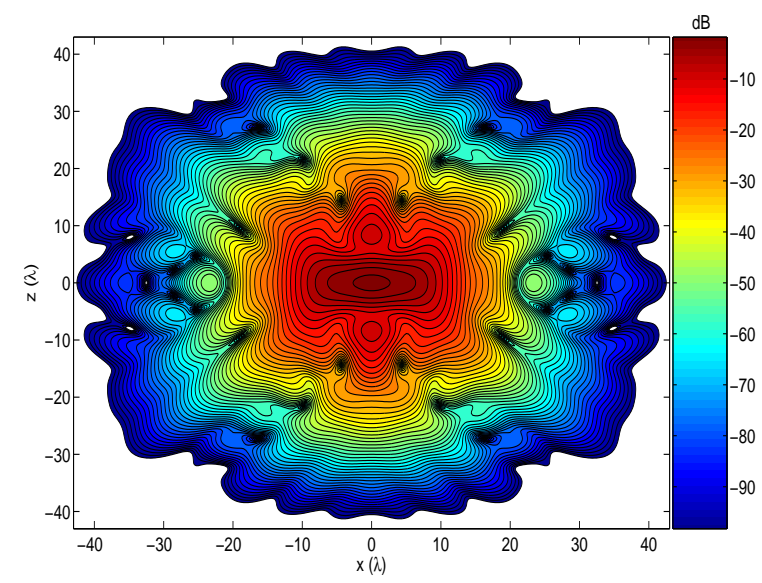

Figure 2: $20 \log _{10}\left|\mathrm{E}_{x}^{s}\right|$.

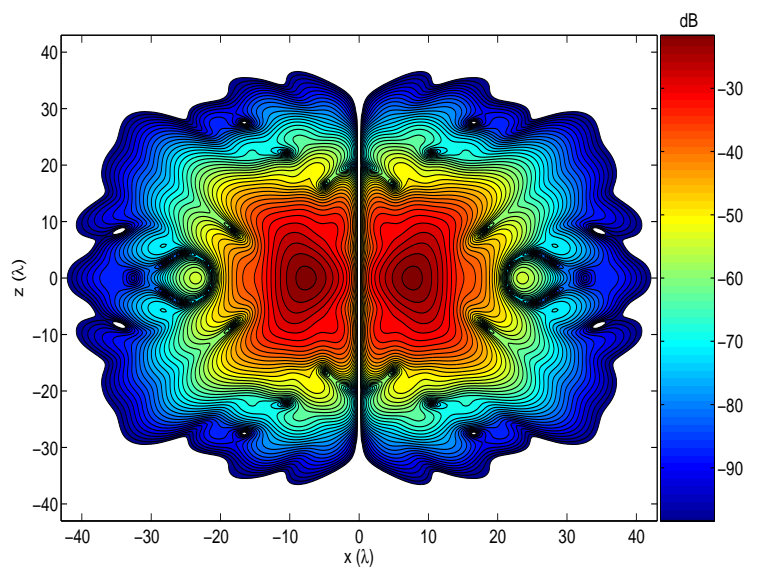

Figure 3: $20 \log _{10}\left|\mathrm{E}_{z}^{s}\right|$.

where the Fourier Transform FT is defined similarly as IFT in (3) and $\triangle S$ is the small surface patch between two adjacent reference cylindrical surfaces; what's more, the following quantities have been defined,

$$
\gamma_{m}\left(\Lambda \rho_{r}\right)=\left.\frac{\pi}{i 2} \frac{\Lambda^{n} \rho^{\prime}}{n !} \frac{\partial^{(n)} H_{m}^{(1)}(\Lambda \rho)}{\partial(\Lambda \rho)^{(n)}}\right|_{\rho_{r}}, \quad \underset{\tilde{\mathbf{J}}_{s}\left(\mathbf{r}^{\prime}\right)}{\tilde{\mathbf{M}}_{s}\left(\mathbf{r}^{\prime}\right)}=\frac{1}{\hat{\mathbf{n}} \cdot \hat{\boldsymbol{\rho}}^{\prime}} \underset{\mathbf{J}_{s}\left(\mathbf{r}^{\prime}\right)}{\mathbf{M}_{s}\left(\mathbf{r}^{\prime}\right)}
$$

$\hat{\mathbf{n}}$ is the surface normal to $S$.

\section{IVT NTTMMERTCA T. R E.STTIT}

To sh

od 1 has been ample

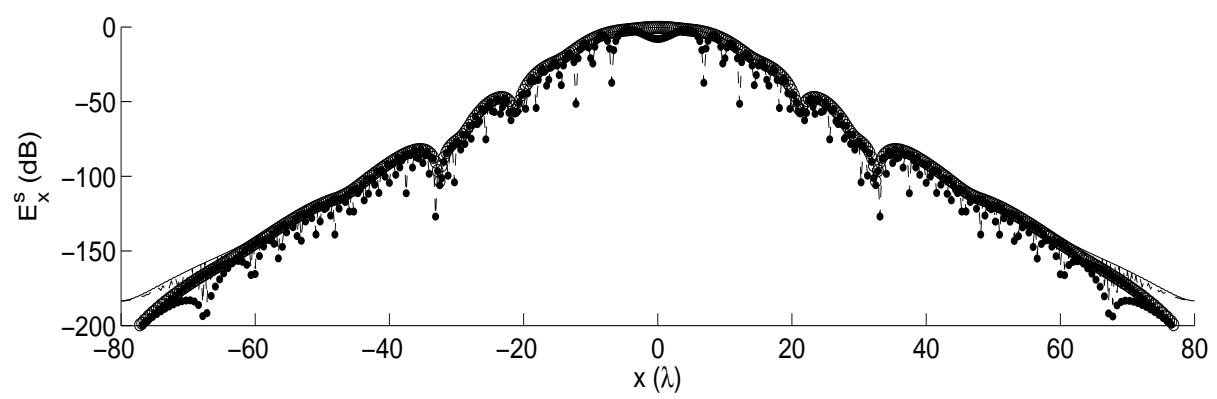

a)

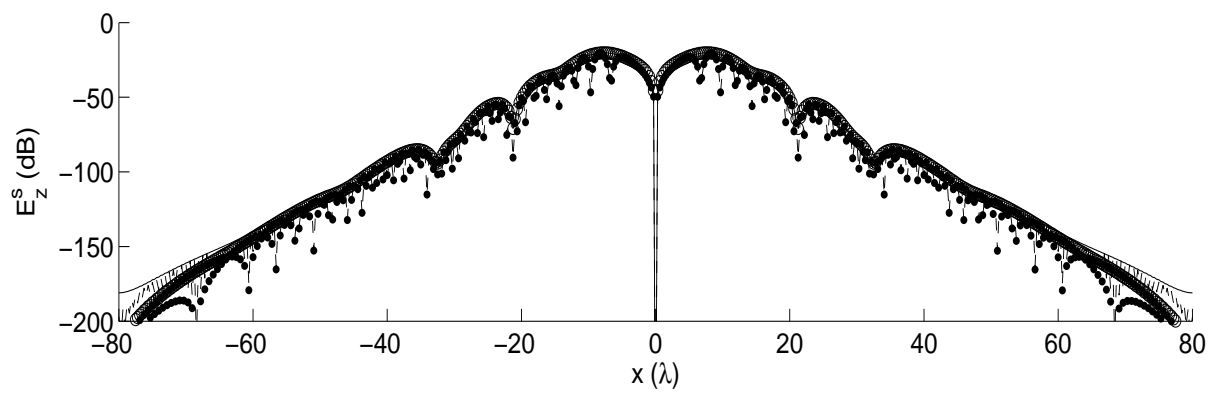

b)

Figure 4: Comparison of the cylindrical TI-FFT algorithm with the direct integration method: a) $\mathrm{E}_{x}(\mathrm{~dB})$; and b) $\mathrm{E}_{z}(\mathrm{~dB})$. Plots are shown in $\hat{\mathbf{x}}$ direction, across the maximum value point of $\left|\mathrm{E}_{x}\right|$. Solid and dashed lines denote the magnitude and real part obtained from the direct integration method respectively; circles and dots denote the magnitude and real part obtained from the cylindrical TI-FFT algorithm respectively. $\mathrm{E}_{y}$ is small and not shown. 


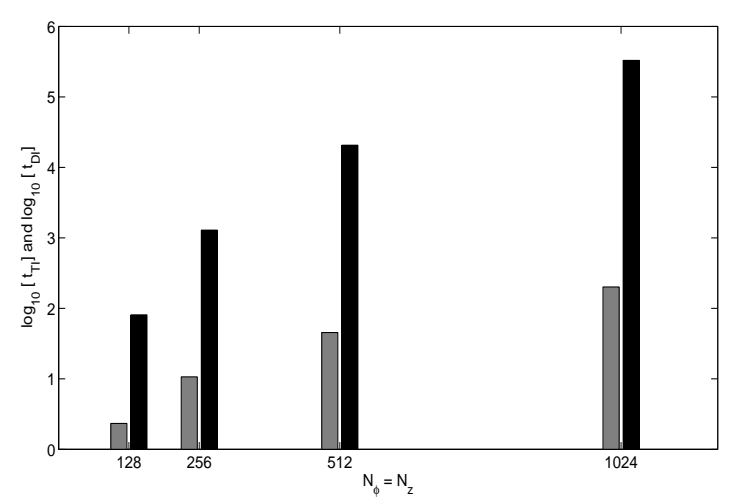

Figure 5: The logarithmic CPU time $\mathrm{t}_{\mathrm{TI}}$ and $\mathrm{t}_{\mathrm{D}]}$

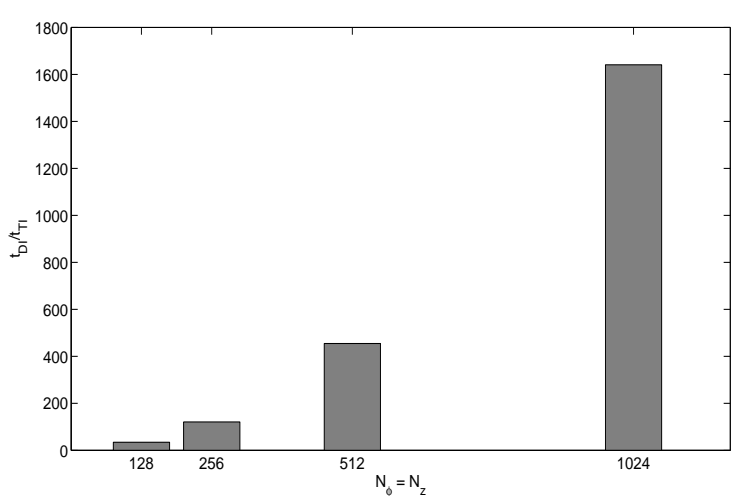

Figure 6: The CPU time ratio of $t_{\mathrm{DI}} / \mathrm{t}_{\mathrm{TI}}$.

used for such purpose is a $110 \mathrm{GHz}(\lambda \sim 2.7 \mathrm{~mm})$ Fundamental Gaussian Beam (FGB) scattered by a PEC quasi-cylindrical surface with a cosine wave perturbation. The incident FGB is $\hat{\mathbf{x}}^{-}$ polarized and propagates at $\hat{\mathbf{z}}$ direction, with symmetrical beam waist radii $w_{x}=w_{y}=8 \lambda$. The quasi-cylindrical PEC surface is given as

$$
\begin{gathered}
y(x, z)=\sqrt{(80 \lambda)^{2}-x^{2}}+0.1 \lambda \cos \left(2 \pi \frac{x}{20 \lambda}\right) \cos \left(2 \pi \frac{z}{20 \lambda}\right) \\
\rho(x, z)=x \cos \phi+y \sin \phi, \quad \phi=\arctan \left[\frac{y}{x}\right]
\end{gathered}
$$

The scattered field $\mathbf{E}^{s}$ is evaluated on plane $y=0$ (where the incident FGB starts to propagate). Fig. 2 and Fig. 3 show the magnitude patterns of the x-component $\mathrm{E}_{x}^{s}$ and the z-component $\mathrm{E}_{z}^{s}$ of the scattered output field $\mathbf{E}^{s}$ (y-component $\mathrm{E}_{y}^{s}$ is small and not shown). The comparison of result obtained from the cylindrical TI-FFT algorithm and that from the direct integration method is given in Fig. 4, for both the magnitude and the real part.

The CPU time for the cylindrical TI-FFT algorithm $t_{\mathrm{TI}}$ and the CPU time for the direct integration method $t_{D I}$ are shown in Fig. 5. The ratio $t_{D I} / t_{T I}$ is shown in Fig. 6, for different size of the computational grid $\left(\mathrm{N}=\mathrm{N}_{\phi} \times \mathrm{N}_{z}\right)$. All work was done in Matlab 7.0.1, on a $1.66 \mathrm{GHz} \mathrm{PC}$, with Intel Core Duo and 512 MB RAM.

\section{CONCLUSION}

The cylindrical TI-FFT algorithm for the computation of the electromagnetic wave propagation and scattering has been introduced for the narrow-band beam and the quasi-geometry geometry.

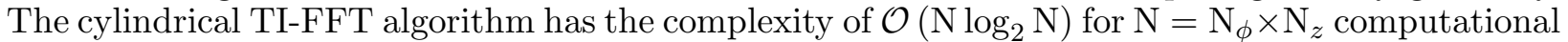
grid. The algorithm allows for a low sampling rate (limited the Nyquist sampling rate) and doesn't have the problem of singularity.

\section{Acknowledgements}

This work was supported by the U.S. Dept. of Energy under the contract DE-FG02-85ER52122.

\section{REFERENCES}

1. Shaolin Liao and R. J. Vernon, "A new fast algorithm for field propagation between arbitrary smooth surfaces", the joint $30^{\text {th }}$ Infrared and Millimeter Waves and $13^{\text {th }}$ International Conference on Terahertz Electronics, Williamsburg, Virginia, USA, 2005, ISBN: 0-7803-9348-1, INSPEC number: 8788764, DOI: 10.1109/ICIMW.2005.1572687, Vol. 2, pp. 606-607.

2. Shaolin Liao and Ronald J. Vernon, "The Near-Field and Far-Field Properties of the Cylindrical Modal Expansions with Application in the Image Theorem," In 2006 Joint 31st International Conference on Infrared Millimeter Waves and 14th International Conference on Teraherz Electronics, pages 260-260, September 2006. ISSN: 2162-2035. 
3. Shaolin Liao and R.J. Vernon, "A new fast algorithm for calculating near-field propagation between arbitrary smooth surfaces," In 2005 Joint 30th International Conference on Infrared and Millimeter Waves and 13th International Conference on Terahertz Electronics, volume 2, pages 606-607 vol. 2, September 2005. ISSN: 2162-2035.

4. Shaolin Liao, Henry Soekmadji, and Ronald J. Vernon, "On Fast Computation of Electromagnetic Wave Propagation through FFT," In 2006 7th International Symposium on Antennas Propagation EM Theory, pages 1-4, October 2006.

5. Shaolin Liao and Ronald J. Vernon, "The Cylindrical Taylor-Interpolation FFT Algorithm," In 2006 Joint 31st International Conference on Infrared Millimeter Waves and 14th International Conference on Teraherz Electronics, pages 259-259, September 2006. ISSN: 2162-2035.

6. Shaolin Liao, "Beam-shaping PEC Mirror Phase Corrector Design," PIERS Online, 3(4):392396, 2007.

7. Shaolin Liao, "Fast Computation of Electromagnetic Wave Propagation and Scattering for Quasi-cylindrical Geometry," PIERS Online, 3(1):96-100, 2007.

8. Shaolin Liao, "On the validity of physical optics for narrow-band beam scattering and diffraction from the open cylindrical surface," Progress in Electromagnetics Research Symposium (PIERS), vol. 3, no. 2, pp. 158162 Mar., 2007. arXiv:physics/3252668. DOI: 10.2529/PIERS060906142312

9. Shaolin Liao, Ronald J. Vernon, and Jeffrey Neilson, "A high-efficiency four-frequency mode converter design with small output angle variation for a step-tunable gyrotron," In 2008 33rd International Conference on Infrared, Millimeter and Terahertz Waves, pages 1-2, September 2008. ISSN: 2162-2035.

10. S. Liao, R. J. Vernon, and J. Neilson, "A four-frequency mode converter with small output angle variation for a step-tunable gyrotron," In Electron Cyclotron Emission and Electron Cyclotron Resonance Heating (EC-15), pages 477-482. WORLD SCIENTIFIC, April 2009.

11. Ronald J. Vernon, "High-Power Microwave Transmission and Mode Conversion Program," Technical Report DOEUW52122, Univ. of Wisconsin, Madison, WI (United States), August 2015.

12. Shaolin Liao, Multi-frequency beam-shaping mirror system design for high-power gyrotrons: theory, algorithms and methods, Ph.D. Thesis, University of Wisconsin at Madison, USA, 2008. AAI3314260 ISBN-13: 9780549633167.

13. Shaolin Liao and Ronald J. Vernon, "A Fast Algorithm for Wave Propagation from a Plane or a Cylindrical Surface," International Journal of Infrared and Millimeter Waves, 28(6):479-490, June 2007.

14. S.-L. Liao and R. J. Vernon, "Sub-THz Beam-Shaping Mirror System Designs for Quasioptical Mode Converters in High-power Gyrotrons," Journal of Electromagnetic Waves and Applications, 21(4):425-439, January 2007. Publisher: Taylor \& Francis.

15. Shaolin Liao, "Miter Bend Mirror Design for Corrugated Waveguides," Progress In Electromagnetics Research, 10:157-162, 2009.

16. Shaolin Liao and Ronald J. Vernon, "A Fast Algorithm for Computation of Electromagnetic Wave Propagation in Half-Space," IEEE Transactions on Antennas and Propagation, 57(7):2068-2075, July 2009.

17. Shaolin Liao, N. Gopalsami, A. Venugopal, A. Heifetz, and A. C. Raptis, "An efficient iterative algorithm for computation of scattering from dielectric objects," Optics Express, 19(4):33043315, February 2011. Publisher: Optical Society of America.

18. Shaolin Liao, "Spectral-domain MOM for Planar Meta-materials of Arbitrary Aperture Waveguide Array," In 2019 IEEE MTT-S International Conference on Numerical Electromagnetic and Multiphysics Modeling and Optimization (NEMO), pages 1-4, May 2019.

19. W. M. Leach, Jr. and D. T. Paris, "Probe-compensated near-field measurements on a cylinder," IEEE Trans. on Antennas and Propagat., Vol. 21, Issue 4, Jul. 1973, pp. 435-445. 